\title{
Silent Ischemic Stroke Was Revealed after Screening for Cognitive Dysfunction in a Hypertensive Patient with New Onset Atrial Fibrillation - Case Report
}

\author{
Tünde Pál1 ${ }^{1}$ Zoltan Preg²*, Enikő Nemes-Nagy², Robert Gabriel Tripon², Beáta Baróti²,
} Márta Germán-Salló2

1. Emergency County Hospital Targu Mures, Romania

2. University of Medicine and Pharmacy Science and Technology of Targu Mures, Romania

\begin{abstract}
Introduction: Hypertension is one of the most important modifiable risk factor related to cognitive decline and dementia. However, screening for cognitive dysfunction is not part of the routine clinical assessment. Case presentation: In this report, we present the case of a 75 year old hypertensive male patient with new-onset atrial fibrillation, admitted to the Cardiovascular Rehabilitation Clinic Târgu Mureș. Apart from the routine clinical assessment, the evaluation of cognitive functions was performed with three different screening instruments which identified cognitive dysfunction. Depressive state was assessed with the shortened 13 items form of the Beck Depression Inventory BDI-13 (BDI-13) and it showed moderate depression which could influence the results of cognitive tests. Detection of cognitive impairment was followed by magnetic resonance imaging, which revealed not only hypertension specific microvascular impairment but also a sequelae of a former stroke in the territory of the left middle cerebral artery and a possible meningioma. Conclusion: Screening for cognitive dysfunction in high-risk hypertensive patients can be easily performed and in several cases like ours, can unmask silent cerebrovascular pathologies, leading to prognostic and therapeutic consequences.
\end{abstract}

Keywords: atrial fibrillation; silent stroke, dementia

Received 15 February 2019 / Accepted 31 July 2019

\section{Introduction}

There are increasing numbers of patients presenting cognitive decline due to ageing population. The prevalence of mild cognitive impairment is estimated to be from $3 \%$ to $42 \%$ over the age of 65 years. There is strong evidence that those with mild cognitive impairment have a greater risk to develop dementia than people without cognitive dysfunction [1]mild cognitive impairment (MCI. The hypothesis that chronic arterial hypertension may contribute to cognitive decline [1] mild cognitive impairment (MCI, has been supported by several notorious studies [2-5]. Cognitive dysfunction can be assessed by several easily manageable tests. Population-based screening is not justified, however it is recommended in high-risk groups [6].

In the present report, we present the case of an elderly hypertensive patient with common symptoms, who underwent detailed investigations, leading us to a more complex diagnosis. This had implications on the prognosis and treatment of our patient.

\section{Case presentation}

In this report, we present the case of a 75 year old male patient, retired driver, known for approximately 15 years with relatively well controlled arterial hypertension. At the time of admission, he was recently diagnosed with atrial

* Correspondence to: Zoltan Preg

E-mail: preg_zoltan@hotmail.com fibrillation (time of onset unknown) and consequently he was admitted to the Cardiovascular Rehabilitation Clinic for cardiovascular evaluation and initiation of anticoagulation therapy. On presentation, he complained of palpitation, dyspnea on effort and memory loss. He was a nonsmoker, obese, casual alcohol consumer. The patient has previously been diagnosed with hypertensive cardiopathy with good systolic left ventricular function, left anterior fascicular block, ventricular extrasystole, mild mitral, aortic and tricuspid valve insufficiency and gonarthrosis. No family history for cardiovascular or cerebrovascular diseases, dementia or other forms of cognitive dysfunction were reported.

Findings on general physical examination were unremarkable, excepting a higher BMI of $30.66 \mathrm{~kg} / \mathrm{m}^{2}$. Heart sounds were irregular, without murmurs, heart rate was $82 \mathrm{bpm}$ with pulse deficit of $15 \mathrm{bpm}$, blood pressure was $130 / 76 \mathrm{mmHg}$, oxygen saturation $98 \%$ on ambiental air. Laboratory investigations showed normal hemogram, blood lipids (total cholesterol $174.8 \mathrm{mg} / \mathrm{dl}$ on statin therapy), uric acid, liver function and normal fasting glucose level, but with impaired glucose tolerance on oral glucose tolerance test. Serum thyroid hormones were also in normal range. No microalbuminuria was detected, however renal function was found slightly decreased (eGFR $68.31 \mathrm{ml} / \mathrm{min} / 1.73 \mathrm{~m} 2$ ). According to guidelines for the management of arterial hypertension, we evaluated target organ damage [7]. Resting ECG showed atrial fibrillation, 
with $65 \mathrm{bpm}$ average rate, intermediate QRS axis, left anterior fascicular block and no significant repolarization abnormalities. Echocardiography revealed concentric left ventricular hypertrophy (dIVS $14.8 \mathrm{~mm}$, dPW $14 \mathrm{~mm}$ ) with good systolic function (LVEF 57\%), multiple, mild (mitral, aortic) degenerative valve disorders and dilated left atria (LA $54.6 \mathrm{~cm}^{2}$ ). Ambulatory blood pressure monitoring was performed under antihypertensive treatment (ACE inhibitor, beta-blocker with vasodilator action), which showed overall mean BP 130/76 $\mathrm{mmHg}$, daytime mean $117 / 68 \mathrm{mmHg}$, nighttime mean $137 / 81 \mathrm{mmHg}$ and reverse dipping pattern (diurnal index -17/-18\%). 24 hour Holter monitoring demonstrated atrial fibrillation during the whole monitoring period with a mean heart rate of 70 bpm and ectopic ventricular beats $(<1 \%)$, no complex arrhythmias, no ST segment deviation, no long RR intervals were found. No significant atherosclerotic plaques were revealed on carotid artery ultrasound (carotid artery IMT $0.83 \mathrm{~mm}$ bilateral). Abdominal ultrasound was normal excepting prostate hyperplasia. Eye examination included retinal microvasculature evaluation. Retinal photography revealed vascular nipping with no further signs of vascular changes due to arterial hypertension. Consequently, using the Wong and Mitchell classification, the patient was diagnosed stage I hypertensive retinopathy. Further modifications were seen in the macular area of the right eye, consisting of pigmentary changes of unknown aetiology, localised at the outer retina - choroidocapillary junction.

Apart from the routine clinical assessment, according to the guideline for the management of arterial hypertension, the evaluation of cognitive function was also performed [7]. We selected three well-accepted cognitive assessment batteries: Mini Mental State Examination (MMSE), the Montreal Cognitive Assessment (MoCA) and the General Practitioner Assessment of Cognition (GPCOG). Depression was also tested, as an influencing factor of cognitive functions, with the shortened 13 items form of Beck depression inventory (BDI-13). The MMSE is the most widely used cognitive test, it is considered to be the gold standard in detection of dementia with a maximum of 30 points, the cut-off value is 24 points [8]. Our patient had difficulties in attention and calculation, recall, repetition, and copy parts. He obtained 21 points, equivalent to mild dementia. The MoCA is also a 30 points test. It is used for detecting vascular mild cognitive impairment [8].

The patient got lower points in visuospatial/executive, attention, language, abstraction parts and no point in delayed recall section. Total obtained score was 21 points (Figure 1). The GPCOG is used in primary care, it consists of two parts (1.Patient examination, 2.Informant interview) [8]. Except the time orientation section, he got no points. Totalizing 1 point, the result meant cognitive impairment. However, it was unnecessary, the patient's wife filled in the second part (informant review needed between 5-8 points [8] ). The result was controversial, according to that the patient had no significant cognitive impairment.
Finally, it turned out the wife was already diagnosed with dementia. According to all used screening instruments, the patient was diagnosed with $\mathrm{CD}$, with the result of 21 points at MMSE, 21 on MOCA, 1 on GPCOG. BDI -13 questionnaire (11 points) demonstrated moderate depression, which could influence our patient's cognitive functions.

In hypertensive patients with cognitive impairment the markers of cerebral microvascular disease (white matter hyperintensities, silent brain infarcts, lacunar infarcts, microbleeds, brain atrophy) could be demonstrated by brain magnetic resonance imaging (MRI), however, due to the costs and accessibility in most cases it is not feasible [7]. Nonetheless, our patient underwent the brain imaging study, which revealed bilateral periventricular white matter hyperintensities, brain atrophy, lacunar infarcts, furthermore a round mass extra-axial location, parietal meningioma on FLAIR MRI sequence (Figure 1; Figure 2). But surprisingly the radiologist also described on FLAIR and T2 MRI a hyperintense lesion, sequelae of a former stroke in the territory of the left middle cerebral artery (Figure 2).

Based upon these findings our patient's SCORE risk category changed from high to very high risk category followed by therapeutic plan: antihypertension treatment was maintained, as BP was on target level, so was beta-blocker, for rhythm control and BP control. Cardioversion was not an option for several reasons: patient's choice, good control of AF symptoms, dilated left atria, unknown AF time of onset. Patient's stroke risk based on $\mathrm{CHA}_{2} \mathrm{DS}_{2}$-VASc risk score was 5 points, HAS-BLED score 2 points, with clear indication for chronic oral anticoagulation therapy for stroke prevention [9]. Oral anticoagulation therapy with vitamin $\mathrm{K}$ antagonist acenocumarol was initiated, with the achievement of therapeutic target (INR 2.14; 2.5), as the patient could not afford non-vitamin K antagonist oral anticoagulants. The patient needed also a good control of risk factors, including dietary changes for weight reduction and control of glycaemia. After discharge, he was refferred to psychiatric evaluation. He was registered in the national dementia program and Memantine was prescribed for dementia treatment.

\section{Discussion}

One of the main target organ damages caused by arterial hypertension is the brain. Stroke, transient ischemic attacks, hypertension-mediated functional and structural alterations in the microvasculature contribute to deterioration of cognitive functions [10]most commonly caused by Alzheimer disease or cerebrovascular factors (vascular dementia. High blood pressure is considered one of the most important modifiable risk factor, besides diabetes, dyslipidemia, obesity, cardio-and cerebrovascular diseases [11]. In addition to, chronic arterial hypertension was also associated with vascular dementia, age-related dementia and Alzheimer disease. The pathophysiological process, however it is not fully elucidated, is considered to be dis- 


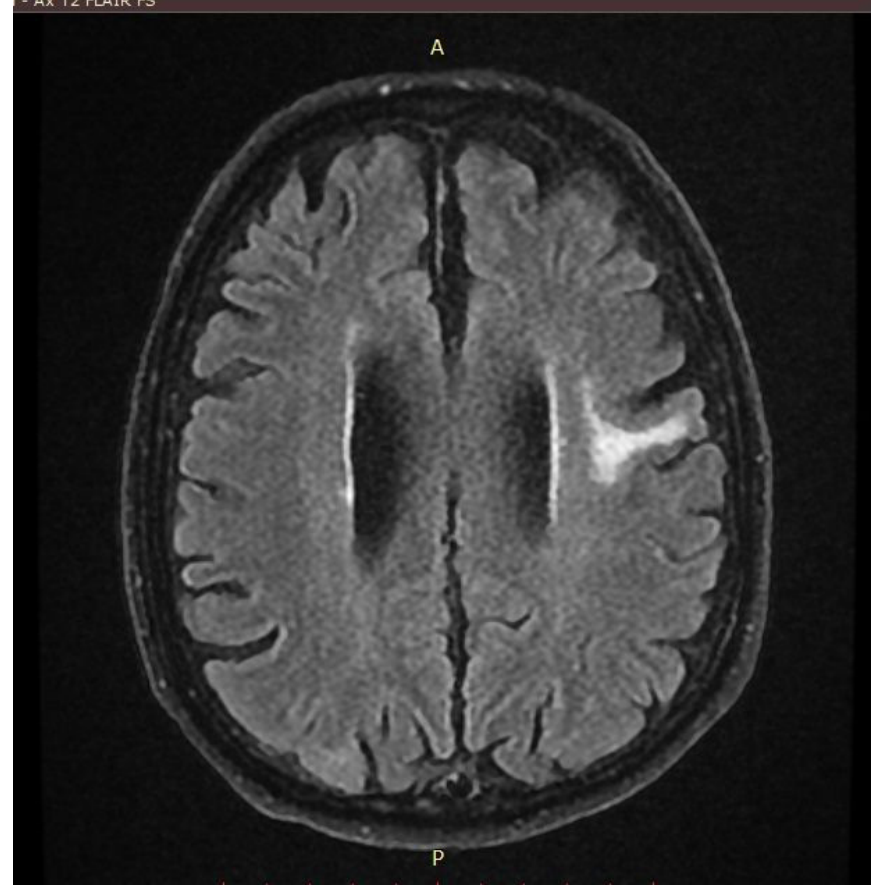

Fig. 1. Bilateral periventricular white matter hyperintensities, significant brain atrophy, hyperintense lesion in the territory of the left middle cerebral artery on FLAIR MRI sequence

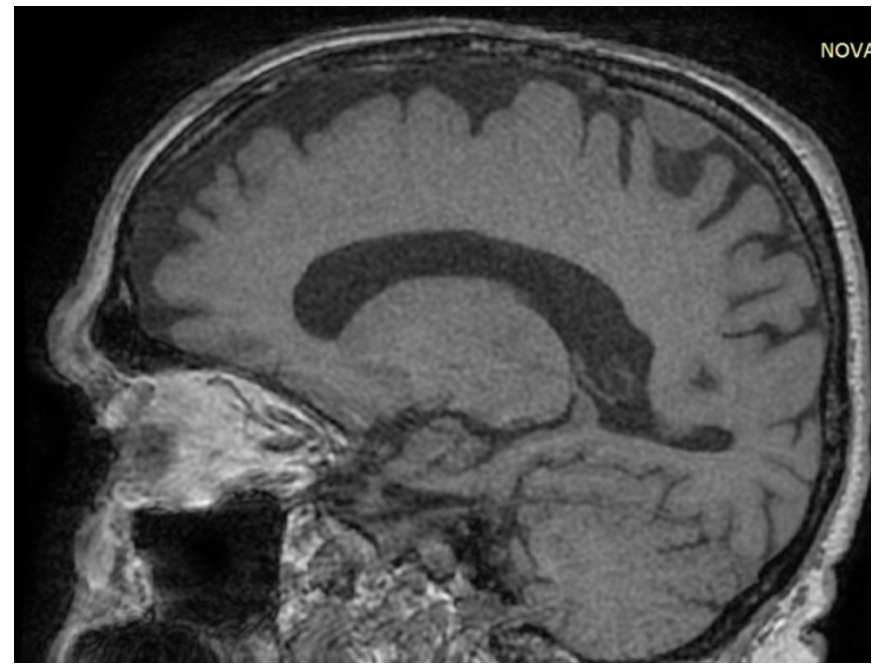

Fig. 2. Cerebral atrophy; round mass extra-axial location, parietal meningioma on FLAIR MRI sequence

ruption of cerebral blood vessels, which activate the process of atherosclerosis, leading to impairment in the cerebrovascular regulatory mechanism. All these alterations lead to cerebral hypoperfusion and structural cerebrovascular changes, like white matter hyperintensities, silent microinfarcts, microbleeds, brain atrophy can be identified with brain magnetic resonance imaging (MRI). Functional changes can be measured by the evaluation of cognitive functions with different tests and questionnaires. Data suggest that the most affected cognitive domains are the executive functions, processing of speed and memory [10] most commonly caused by Alzheimer disease or cerebrovascular factors (vascular dementia. The current guideline for the management of arterial hypertension made a suggestion for the evaluation of cognitive functions as part of the clinical assessment in hypertensive patients with suspicion of cognitive alteration [7].

In the present case, cognitive performances were evaluated owing to the patient's symptoms and as part of a CD screening study amongst all hypertensive inpatients. We identified cognitive dysfunction with three different wellaccepted screening batteries. Interpretation of one of the tests (GPCOG) was deeply influenced by the coincidence, that the patient's wife was already diagnosed with dementia. Radioimaging of the brain revealed the markers of microvascular brain disease, which could explain the poor cognitive performance. In addition silent ischemic stroke was also found on MRI. Silent brain infarct is a common incidental finding on brain imaging, it is much more frequent than clinical stroke and is also associated with an increased risk for decline of cognitive performance and dementia [12]. The MRI findings of this case are similar to those abnormalities which can be observed in chronic hypertensive patients. Silent brain infarcts appear more commonly amongst patients with advanced age, arterial hypertension, diabetes mellitus, and smoking [13]. The prevalence of post-stroke dementia is about 30\% [14,15], however, it differs in countries and regions being mostly measured by MMSE [16]which varies for the difference between the countries, the races, and the diagnostic criteria. The risk of post-stroke cognitive impairment is related to both the demographic factors like age, education and occupation and vascular factors. The underlying mechanisms of post-stroke cognitive impairment are not known in detail. However, the neuroanatomical lesions caused by the stroke on strategic areas such as the hippocampus and the white matter lesions (WMLs. Overall, this accidental discovery in our case moved our patient to the highest cardiovascular risk category, worsening his prognosis. Furthermore, cognitive evaluation in this patient revealed another complication with important treatment repercussion, which included specific treatment for dementia and changes in target levels regarding his cholesterol.

Although, atrial fibrillation was first documented one week before admission, the presence of dilated left atria suggests the long-standing state of it. The particularity of this case is the presence of atrial fibrillation, which can be an etiologic cause of stroke. Data showed that atrial fibrillation increases the risk of stroke and is associated with deterioration of cognitive functions, hence atrial fibrillation indirectly is related to cognitive impairment. Also there is growing evidence, that atrial fibrillation itself can be a risk factor for worsening of cognitive performance [17]. In the Rotterdam cross-sectional study the prevalence of dementia was doubled among patients with AF compared to controls [18]population-based study in the elderly. Methods Of the 6584 participants in the Rotterdam Study aged 55 to 106 years, detailed information on dementia status and ECG abnormalities was available. Dementia was diagnosed in three phases. First, participants were screened. Screenpositive subjects were tested further. Those with possible 
dementia underwent an extensive diagnostic workup. Dementia and dementia subtypes were diagnosed according to prevailing criteria. Cognitive impairment was defined as a Mini-Mental State Examination test score of \&amp;lt;26 points for a nondemented subject. Results Atrial fibrillation was diagnosed in 195 , dementia in 276 , and cognitive impairment in 635 subjects. We found significant positive associations of atrial fibrillation with both dementia and impaired cognitive function (age- and sex-adjusted odds ratios, 2.3 [95\% confidence interval, 1.4 to 3.7$]$ and 1.7 [95\% confidence interval, 1.2 to 2.5 ].

Anticoagulation therapy can be challenging in patients with cognitive dysfunction. This problem was enhanced by the presence of the spouse's dementia. In this case, new oral anticoagulants would have been a better choice. Unfortunately, our patient could not afford this, due to low family income. For this patient would have been important if the insurance system supported the cost of a new oral anticoagulants. Still, our patient needs a closer follow-up by the family physician and cardiologist.

Our case also highlights the importance of early detection of atrial fibrillation. Patients detected with persistent atrial fibrillation may have had several asymptomatic episodes of paroxysmal atrial fibrillation which may have also been complicated with asymptomatic cerebral microembolization, as in our patient. For this reason besides invasive monitoring devices, new approaches for atrial fibrillation detection are needed, as smart watches, mobile phone applications, smart dresses, or wareable fitness trackers [19] .

Overall, we can stipulate that bedside tests like MMSE and MoCA provide an easy screening opportunity in this particular group of patients and can have repercussions in the diagnosis and treatment approach. In the light of all the above mentioned risk factors in the literature in association with cognitive impairment, it makes difficult to determine their independent role in our patient's cognitive deterioration. However, as the population ages, the management of cognitively altered and demented patients is going to be a public issue due to a socioeconomic burden. Prevention would be the most cost-effective method through identification and proper treatment of existing risk factors. Definitely, arterial hypertension is the most investigated risk factor in this field. Emerging evidence proves the phrase of midlife hypertension late life cognitive dysfunction. Data shows that $\mathrm{BP}$ control in midlife in patients without cognitive impairment at baseline, may diminish the risk of late life cognitive dysfunction, in particularly, in processing speed and executive function, which are considered to be the most affected cognitive domains by arterial hypertension. Long-term outcome should be maintaining a good quality of life as long as possible.

\section{Conclusion}

In the present report, we would like to highlight the importance of cognitive assessment in hypertensive patients. Screening for cognitive dysfunction in high-risk hyperten- sive patients can be easily performed. It can unmask silent cerebrovascular pathologies, having prognostic and therapeutic consequences.

\section{Authors' contribution}

Tünde Pál (Conceptualization; Data curation; Formal analysis; Investigation; Writing -original draft)

Zoltan Preg (Conceptualization; Data curation; Formal analysis; Investigation; Methodology; Supervision; Writing - original draft; Writing - review \& editing)

Enikő Nemes-Nagy (Conceptualization; Data curation; Formal analysis; Investigation; Resources; Supervision; Writing - review \& editing)

Robert Gabriel Tripon (Conceptualization; Data curation; Formal analysis; Methodology; Writing - review \& editing)

Beáta Baróti (Data curation; Formal analysis; Investigation; Methodology; Supervision)

Márta Germán-Salló (Conceptualization; Data curation; Funding acquisition; Investigation; Methodology; Project administration; Resources; Supervision; Validation; Writing - review \& editing)

\section{Funding}

Funding was provided by the Hungarian Academy of Science, contract nr. 0346/26.02.2016. Special thanks to the Studium Prospero Foundation.

\section{Conflict of interest}

None to declare.

\section{References}

1. Elias MF, Goodell AL, Dore GA. Hypertension and cognitive functioning: A perspective in historical context. Hypertension. 2012;60(2):260-8.

2. Kilander L, Nyman H, Boberg M, et al. A 20-Year Follow-up of 999 Men. Hypertension. 1998;31:780-7.

3. Elias PK, Elias MF, D'Agostino RB, et al. NIDDM and blood pressure as risk factors for poor cognitive performance: The Framingham Study. Diabetes Care.1997;20(9):1388-95.

4. Stewart R, Xue QL, Masaki K, et al. Change in blood pressure and incident dementia: A 32-year prospective study. Hypertension. 2009;54(2):23340.

5. Gelber PR, Launer JL, White LR. The honolulu-asia aging study: Epidemiologic and neuropathologic research on cognitive impairment. Curr Alzheimer Res.2012;9(6):664-72.

6. Lin JS, O'Connor E, Rossom RC. Screening for Cognitive Impairment in Older Adults: A Systematic Review for the U.S. Preventive Services Task Force. Ann Intern Med. 2013;159(9).

7. Mancia G, Spiering W, Rosei EA, et al. 2018 ESC/ESH Guidelines for the management of arterial hypertension The Task Force for the management of arterial hypertension of the European Society of Cardiology (ESC) and the European Society of Hypertension (ESH). Eur Heart J.2018; 30213104.

8. Larner A. Cognitive Screening Instruments. A practical approach. Liverpool. Springer. 2013;15-17, 111-114, 201-202.

9. Kirchhof P, Benussi S, Kotecha D, et al. 2016 ESC Guidelines for the management of atrial fibrillation developed in collaboration with EACTS. Eur Heart J. 2016;37(38):2893-962.

10. ladecola C, Yaffe K, Biller J, et al. Impact of Hypertension on Cognitive Function: A Scientific Statement from the American Heart Association. Hypertension. 2016;68(6):67-94.

11. Gorelick PB, Scuteri A, Black SE, et al. Vascular contributions to cognitive impairment and dementia: A statement for healthcare professionals from the American Heart Association/American Stroke Association. Stroke. 2011;42(9):2672-713. 
12. Vermeer SH, Prins ND, den Heijer T, et al. Silent Brain Infarcts and the Risk of Dementia and Cognitive Decline. N Engl J Med. 2003;1215-22.

13. Smith EE, Saposnik G, Biessels GJ, et al. Prevention of Stroke in Patients with Silent Cerebrovascular Disease: A Scientific Statement for Healthcare Professionals from the American Heart Association/American Stroke Association. Stroke. 2017;48, 44-71.

14. Hénon H, Pasquier F, Leys D. Poststroke dementia. Cerebrovasc Dis. 2006;22:61-70.

15. Douiri A, Rudd AG, Wolfe CDA. Prevalence of poststroke cognitive impairment: South London stroke register 1995-2010. Stroke.
2013;44(1):138-45.

16. Sun J-H, Tan L, Yu J-T. Post-stroke cognitive impairment: epidemiology, mechanisms and management. Ann Transl Med. 2014;2(8):80.

17. Knecht S, Oelschläger $\mathrm{C}$, Duning $\mathrm{T}$, et al. Atrial fibrillation in strokefree patients is associated with memory impairment and hippocampal atrophy. Eur Heart J. 2008;29(17):2125-32.

18. Ott A, Breteler MMB, de Bruyne MC, et al. A. Atrial Fibrillation and Dementia in a Population-Based Study. Stroke. 1997;28(2):316-321.

19. Hendriks JM, Gallagher C, Middeldorp ME, et al. New approaches to detection of atrial fibrillation. Heart. 2018;0:1-2. 